\title{
Effect of a High-Fat Diet and Metformin on Placental mTOR Signaling in Mice
}

\author{
Matthew R. Grace, MD ${ }^{1}$ Sarah K. Dotters-Katz, MD² \\ Kim Boggess, $\mathrm{MD}^{4}$ Victoria Bae-Jump, MD, $\mathrm{PhD}^{3}$ \\ ${ }^{1}$ Tennessee Maternal Fetal Medicine and the University of Tennessee, \\ Division of Obstetrics and Gynecology, Department of Clinical \\ Medicine Education, Nashville, Tennessee \\ 2 Division of Maternal Fetal Medicine, Department of Obstetrics and \\ Gynecology, Duke University, Durham, North Carolina \\ ${ }^{3}$ Division of Gynecologic Oncology, University of North Carolina \\ School of Medicine, Chapel Hill, North Carolina \\ ${ }^{4}$ Division of Maternal-Fetal Medicine, Department of Obstetrics and \\ Gynecology, University of North Carolina School of Medicine, Chapel \\ Hill, North Carolina \\ ${ }^{5}$ Division of Maternal-Fetal Medicine, Department of Obstetrics and \\ Gynecology and Carolina Institute for Environmental Health \\ Solutions, University of North Carolina School of Medicine, \\ Chapel Hill, North Carolina
}

Am J Perinatol Rep 2019;9:e138-e143.
Chunxiao Zhou, MD, PhD 3 Tracy Manuck, MD ${ }^{4,5}$

Address for correspondence Matthew R. Grace, MD, Tennessee Maternal Fetal Medicine and the University of Tennessee Health Sciences Center, 201 23rd Avenue, North, Nashville, TN 37203 (e-mail: mgrace@tnmfm.com).

\begin{abstract}
Keywords

- metformin

- mTOR

- mice

- high-fat diet
\end{abstract}

Obesity and excessive gestational weight gain (GWG) in pregnancy result in adverse maternal and fetal outcomes and significant health costs. ${ }^{1-3}$ Compared with normal weight women, obese women are at increased risk for pregnancy loss, gestational diabetes, preeclampsia, and still- birth, ${ }^{1,2,4,5}$ and their infants are at increased risk of macrosomia, being large for gestational age, birth trauma, childhood obesity, and metabolic syndrome. ${ }^{6-9}$ In preclinical animal models, dam consumption of a high-fat diet (HFD) results in excessive weight gain, increased fetal growth, received

October 25, 2018

accepted after revision

December 11, 2018
DOI https://doi.org/

10.1055/s-0039-1683362. ISSN 2157-6998.
Copyright $\odot 2019$ by Thieme Medical

Publishers, Inc., 333 Seventh Avenue, New York, NY 10001, USA. Tel: +1(212) 584-4662.
License terms

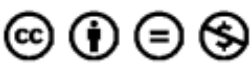


inflammation, and altered maternal and fetal metabolic programming. ${ }^{10-12}$ In human studies, obese women are more likely to have excessive GWG which makes it difficult to separate the effects of baseline maternal obesity from the effects of excessive GWG on offspring birth weight, metabolic programming, and adverse maternal or infant outcomes.

The increase in the prevalence of both maternal obesity and excessive GWG and their deleterious fetal effects have led researchers to seek interventions to limit excess maternal weight gain, fetal overgrowth, and adverse fetal metabolic programming. One such intervention tested is metformin, a biguanide oral hypoglycemic agent used as a first-line agent to treat type II diabetes mellitus. ${ }^{13}$ In addition to its glucose-lowering effects, metformin reduces weight gain, systemic inflammation, and inhibits the mechanistic target of rapamycin (mTOR) pathway, a nutrient-sensing protein complex implicated in diseases where growth and homeostasis are compromised, such as obesity, cancer, and diabetes. ${ }^{14-17}$ mTOR functions as a part of two protein signaling complexes, mTORC1 and mTORC2. mTORC1 is activated by growth factors such as insulin via the insulin/Akt pathway ( - Fig. 1). Activation of mTORC1 results in phosphorylation of S6 (p-S6) which subsequently results in cellular translation and cell growth. ${ }^{18}$ Metformin is thought to inhibit mTORC1. ${ }^{19}$ We previously reported that when pregnant mice were exposed to an HFD during pregnancy, they gained more weight and had heavier fetuses compared with those fed a control diet (CD). ${ }^{20}$ In our model, metformin attenuated fetal weight gain in HFDfed dams compared with dams fed a control diet. While its ability to inhibit mTOR pathway signaling in animal tissues and numerous cancers have been well-described, ${ }^{19,21-23}$ metformin's effect on placental mTOR is unknown. Placen- tal mTOR inhibition may be a mechanism by which metformin attenuates fetal weight gain. Our objective was to measure the effects of an HFD with and without concomitant metformin exposure on placental mTOR signaling. We hypothesized that among HFD-dams, metformin inhibits placental mTOR signaling among placentas exposed to a maternal high-fat diet compared with placentas exposed to a maternal $\mathrm{CD}$.

\section{Materials and Methods}

\section{Animal Model}

The Institutional Animal Care and Use Committee (IACUC) at our institution approved this protocol (15-163.0) and all experimental animals were maintained in accordance with the IACUC and the National Institutes of Health $(\mathrm{NIH})$ guide for the care and use of laboratory animals.

Our model has been described in detail previously. ${ }^{20}$ Briefly, female FVB (friend virus B)-strain mice $(n=20)$ were weighed and then bred with control FVB males. On embryonic day (e) 0.5 (defined upon the detection of a vaginal plug) pregnant dams were allocated into one of four diet and treatment groups ( $n=5$ dams per group); group 1: CD (10\% calories from fat, Research Diets, New Brunswick, NJ) + control treatment (CT, water). Group 2: $\mathrm{CD}+$ metformin (MT) in water $(2.5 \mathrm{mg} / \mathrm{mL}){ }^{24,25}$ Group 3: HFD (60\% calories from fat, Research Diets, New Brunswick, $\mathrm{NJ})+$ CT. Group 4: HFD + MT in water $(2.5 \mathrm{mg} / \mathrm{mL})$. All groups were fed ad libitum until euthanasia.

On e17.5 dams were anesthetized with ketamine and the dams were euthanized via cervical dislocation. Fetuses and placentas were immediately removed from the uterine horns, weighed, and frozen at $-80^{\circ} \mathrm{C}$.

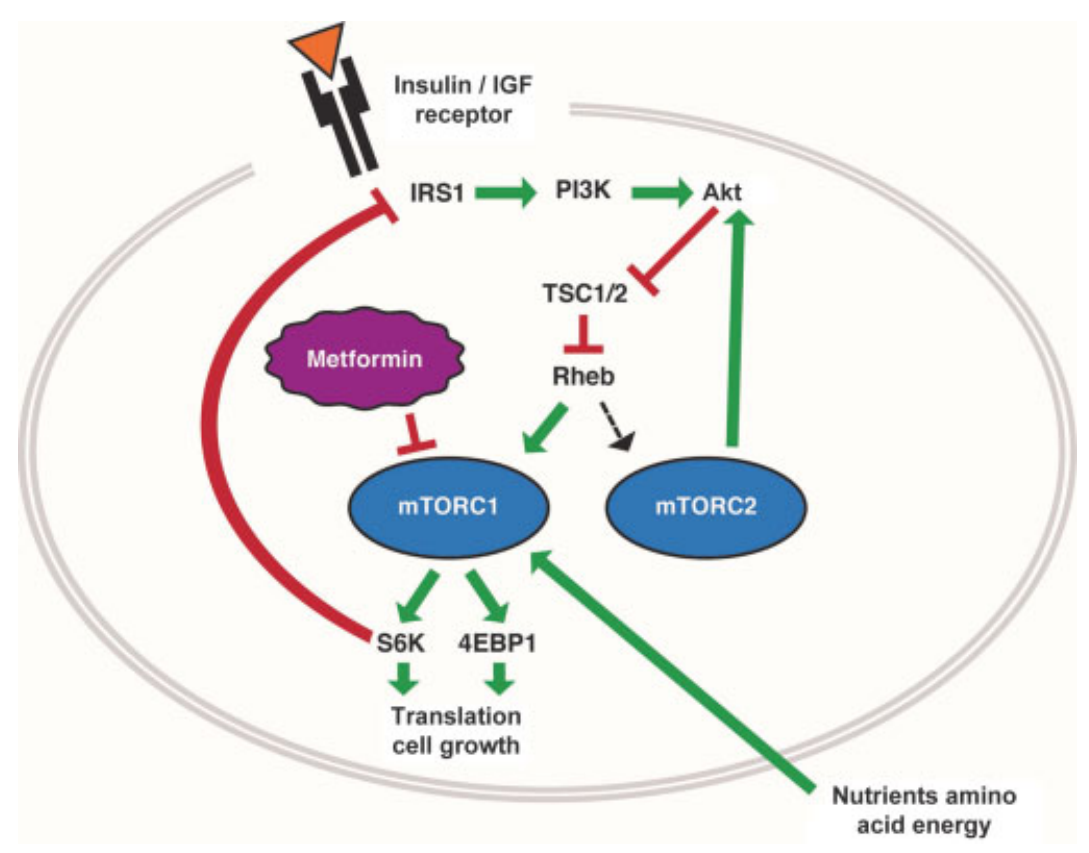

Fig. 1 Model illustrating mTOR signaling. IGF, insulin-like growth factor; IRS1, insulin receptor substrate 1; PI3K, phosphoinositide 3-kinase; TSC1/2, tuberous sclerosis 1 and tuberous sclerosis 2; Rheb, rhas homolog enhanced in brain; mTORC1/2, mechanistic target of rapamycin complex 1 and 2; S-6K, S-6 kinase; 4EBP1, eukaryotic translation initiation factor 4e binding protein 1. 


\section{Western Blot}

Five placentas selected from each treatment group (one per treatment dam) were prepared in Tissues PE LB buffer + PhosStop following manufacturer instructions. Lysate protein concentrations were measured using a BCA Protein Assay Kit (Pierce, Rockford, IL). Equal amount of protein $(50 \mu \mathrm{g})$ was separated by gel electrophoresis and transferred onto a PVDF membrane. The membrane was blocked with 5\% nonfat dry milk and then incubated with a 1:1,000 dilution of primary antibody (phos-Akt and phos-S6-kinase, Cell Signaling Technology, Danvers, MA) overnight at $4^{\circ} \mathrm{C}$. We selected phos-Akt and phos-S6 kinase because phos-Akt expression provides insight into upstream activation and feedback of the mTOR pathway and phos-S6 kinase provides insight into downstream activation of the pathway (- Fig. 1).26,27 The membrane was then washed and incubated with a secondary peroxidase conjugated antibody for 1 hour after washing. Antibody binding was detected using an enhanced chemiluminescence detection buffer by Alpha Innotech imaging system (San Leandro, CA). All blots were stripped and reprobed with pan-Akt, pan-S6-k, and either anti- $\alpha$-tubulin or anti- $\beta$-actin. Blots were imaged and densities were measured using Image Laboratory software (Bio-Rad Laboratories, Inc., Hercules, CA). Western blot results are reported as relative expression (compared with the reference protein $\alpha$-tubulin or $\beta$-actin). Analyses were performed using STATA version 14.2 (College Station, TX). Analysis of variance (ANOVA) and pairwise Student's $t$-tests were used to compare continuous parametric outcomes. A $p$-value of $<0.05$ was considered significant.

\section{Results}

Characteristics of the dams, fetuses, and placentas in the study are reported in - Table 1. Dam mean starting weight, litter size, and placenta weight were not significantly different among treatment groups. We have previously reported that compared with control diet-fed dams (group 1), HFD-fed dams (group 3) gained significantly more weight during pregnancy. Among HFD-fed dams, there were no significant differences in dam weight gain between control (group 3) and metformin-treated dams (group 4). Additionally, compared with control diet-fed dams (group 1), HFD-fed dams (group 3) had significantly heavier. In HFD-fed dams, compared with control-treated (group 3), the metformin-treated (group 4) had significantly lower fetal weight. ${ }^{* *}$ Neeta Reference.

-Fig. 2 shows placental p-Akt Western blot results by treatment group. Compared with CD-fed dams, HFD-fed dams had significantly higher placental p-Akt protein expression $(p<0.001)$. Metformin did not decrease placental p-Akt expression among HFD-fed dams. Rather, compared with control-treated dams receiving an HFD, placental Akt was significantly higher in metformin-treated dams receiving an HFD ( $p=0.004)$.

-Fig. 3 shows placental p-S6 western blot results. Among $\mathrm{CD}$-fed dams, there was no significant difference in placental p-S6 expression in metformin versus control-treated groups. Among HFD-fed dams; however, placental p-S6 expression was lower in those exposed to metformin versus control $(p=0.001)$.

\section{Comment}

In our mouse model, we found that feeding pregnant dams an HFD increased placental expression of p-Akt, an activity marker of the nutrient-sensing pathway mTOR. We also found that in-utero metformin exposure attenuated HFDinduced placental p-Akt expression, and decreased placental p-S6 expression.

We chose to look at placental protein concentrations of p-S6 and p-Akt as they represent markers of MTORC1 activity and signaling. P-S6 is often used as a downstream marker of mTORC1 activity since it is specifically phosphorylated by mTORC1 both in vitro and in vivo. ${ }^{28}$ Akt is a serine/threonine kinase that acts downstream of growth factors, such as insulin, and upstream of mTORC1. Akt increases signaling of mTORC1 but there are multiple feedback mechanisms to Akt, including inhibitory feedback via p-S6 and activating feedback via mTORC2. ${ }^{29}$ mTOR is present in human and animal placentas and placental mTOR dysregulation is implicated in animal models of fetal growth restriction and overgrowth. ${ }^{30,31}$ Metformin's effects on placental mTOR signaling as measured by concentrations of these markers are unknown.

It is plausible that in our study the HFD increased placental p-Akt expression via upregulation of insulin/insulin growth factor-1 receptor signaling and that metformin (which inhibits mTORC1) exposure further increased p-Akt expression via mTORC2 (-Fig. 1). We hypothesized that an

Table 1 Dam weight, litter size, and placental weight characteristics by treatment group

\begin{tabular}{|l|l|l|l|l|}
\hline & $\begin{array}{l}\text { Group1 (CD + CT) } \\
\boldsymbol{n}=5\end{array}$ & $\begin{array}{l}\text { Group 2 (CD + MT) } \\
\boldsymbol{n}=5\end{array}$ & $\begin{array}{l}\text { Group 3 (HFD + CT) } \\
\boldsymbol{n}=5\end{array}$ & $\begin{array}{l}\text { Group 4 (HFD + MT) } \\
\boldsymbol{n}=5\end{array}$ \\
\hline $\begin{array}{l}\text { Dam starting weight, } \\
\text { g (mean } \pm \text { SEM) }\end{array}$ & $23.2(0.34)$ & $23.0(0.39)$ & $22.2(0.58)$ & $24.4(0.93)$ \\
\hline $\begin{array}{l}\text { Litter Size, } \\
n \text { (median } \pm \text { IQR) }\end{array}$ & $11(11-11)$ & $9(9-9)$ & $10(9-10)$ & $10(9-11)$ \\
\hline $\begin{array}{l}\text { Mean placenta weight, } \\
\text { g(mean } \pm \text { SEM) }\end{array}$ & $0.088(0.010)$ & $0.072(0.003)$ & $0.10(0.004)$ & $0.092(0.005)$ \\
\hline
\end{tabular}

Abbreviations: CD, control diet; HFD, high-fat diet; CT, control; MT, metformin; SEM, standard error of the mean; IQR, interquartile range (compared with pairwise Student's $t$-test). 


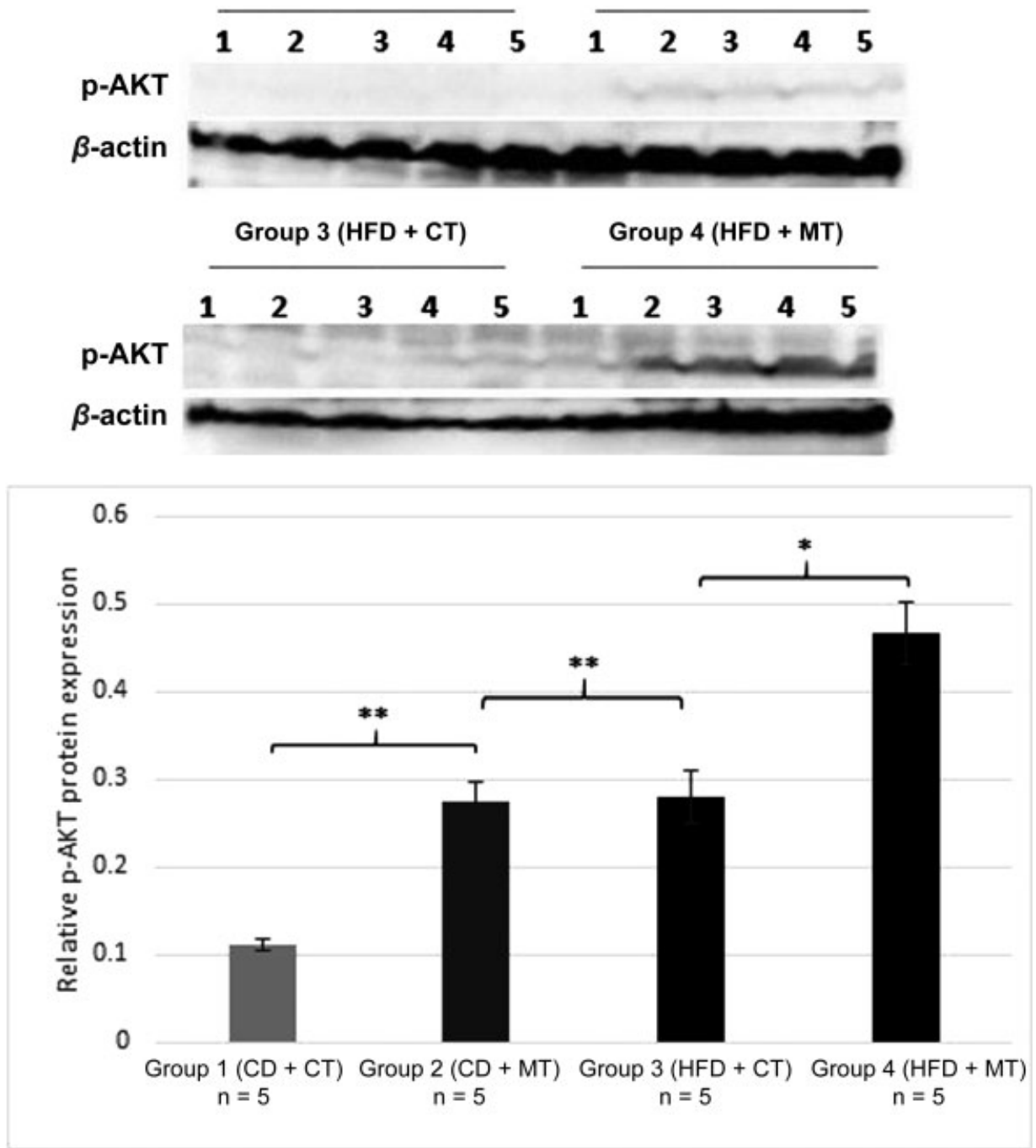

Fig. 2 Western blot analysis of placental expression of p-Akt, by treatment group. ${ }^{*} p<0.001,{ }^{* *} p<0.0001$. CD, control diet; CT, control treatment; HFD, high-fat diet; MT, metformin treatment.

HFD would increase p-S6, a downstream product of mTORC1 which is responsible for increased protein translation and cell growth. P-S6, however, was not increased among control treatment mice when an HFD was added. Thus, in our model, increase in fetal weight is not associated with the increased mTORC1 signaling as a result of the HFD exposure. Among dams exposed to an HFD, however, p-S6 was decreased as a result of metformin exposure. This suggests that metformin inhibition of MTORC1 signaling only occurred in the presence of an HFD exposure.

We hypothesized that an HFD alone would increase downstream markers of mTOR (p-S6) based upon other work that showed that maternal diabetes promoted downstream mTOR signaling in rabbit preimplantation embryos. ${ }^{30}$ Our finding that an HFD alone did not increase placental p-S6 expression may be due to differences in the models used (HFD vs. diabetic model), tissues studied (placenta vs. embryo), and duration of the exposures (17.5 days). mTORC1 receives inputs from at least five extraand intracellular signals: growth factors, stress, energy status, oxygen, and amino acids. ${ }^{17}$ It's possible that an HFD for 17.5 days was not sufficient (either in terms of the diet composition or the duration of the exposure) to significantly affect mTOR1 signaling.

Our animal findings are in contrast to results from a recent double-blind, placebo-controlled randomized trial of metformin versus placebo in obese pregnant women. In this trial, compared with placebo, metformin resulted in less maternal weight gain but no significant differences in neonatal birth weight. ${ }^{16}$ Explanations for this difference may be a lack of control of dietary intake in the clinical trial and effects of other environmental exposures and dietary exposures that were not measured in the trial. Additionally, the trial enrolled women with a body mass index $>35$, and thus the metabolic exposure was obesity and not a high-fat diet or excessive GWG.

Our study has several strengths. We were able to measure the effects of an HFD and metformin in well-timed and characterized pregnancies. The diets that the mice received were standardized. We were also able to demonstrate that an 

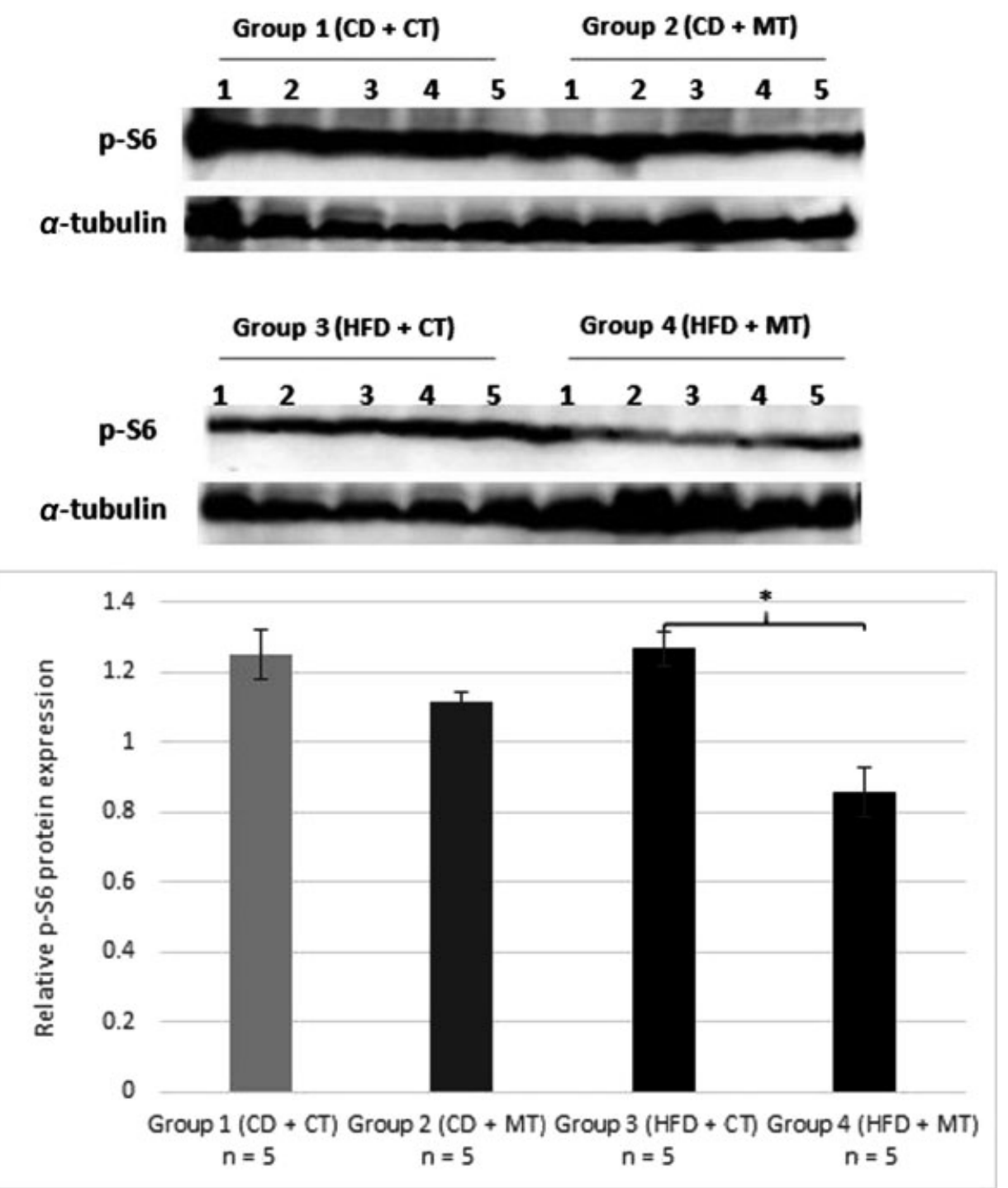

Fig. 3 Western blot analysis of placental expression of $\mathrm{p}-\mathrm{S} 6$, by treatment group. ${ }^{*} p=0.001 ; \mathrm{CD}$, control diet; $\mathrm{CT}$, control treatment; $\mathrm{HFD}$, high-fat diet; MT, metformin treatment.

HFD exposure that began at the onset of pregnancy significantly increased dam and fetal weight without the confounding effects of maternal obesity.

The limitations of our study include that it was not a diabetes or obesity model which may have more direct clinical application. This may have limited our ability to detect differences in mTOR signaling as a result of a chronic obesity exposure. In addition, an HFD may affect and activate mTOR signaling differently than a diet high in sugar, protein, or total calories. Finally, the number of dams and placentas from each group (five) may have limited our ability to detect differences in dam weight gain or mTOR signaling as a result of metformin exposure.

Metformin is widely prescribed in pregnancy to treat both pre-existing diabetes and gestational diabetes. It is generally thought to be safe but few randomized trials have assessed its short-term and long-term effects on offspring. The possibility that it may augment placental mTOR signaling as a result of different maternal metabolic exposures warrants additional research on its effects in pregnancy.

\section{Conclusion}

In conclusion, in our mouse model, placental expression of $\mathrm{p}$ Akt was increased as a result of both HFD and metformin exposures. Among control treatment mice, placental p-S6 expression was similar between $\mathrm{CD}$ and HFD-fed mice. However, placental p-S6 expression was decreased in those placentas exposed to both an HFD and metformin, compared with those exposed to HFD alone. This pattern suggests that increases in fetal weight are not associated with increased mTORC1 signaling as a result of the HFD exposure. Among dams exposed to an HFD, however, p-S6 was decreased as a result of metformin exposure. This suggests that metformin inhibition of mTORC1 signaling only occurred in the presence of an HFD exposure. We theorize that in this model, an HFD did not activate mTOR pathway signaling but metformin appears to have inhibited mTOR pathway signaling in the presence of an HFD and, in doing so, reduced fetal weight. ${ }^{20}$ As the nutrient-sensing capabilities of placental mTOR and its effects on fetal growth continue to be studied, the role that 
metformin may play as a placental mTOR pathway inhibitor should continue to be explored.

\section{Presentation}

Presented, in part, in poster format at the Society for Maternal Fetal Medicine's 37th Annual Pregnancy Meeting, January 27, 2017, Las Vegas, NV.

\section{Financial Disclosure}

This study was funded, in part, by the UNC Cefalo-Bowes Young Investigator Award (Dr. M.R.G.), the UNC Oliver Smithies Investigator Award (Dr. K.B.).

\section{Conflict of Interest}

The authors report no conflicts of interest.

\section{References}

1 Anderson NH, McCowan LM, Fyfe EM, et al; SCOPE Consortium. The impact of maternal body mass index on the phenotype of preeclampsia: a prospective cohort study. BJOG 2012;119(05):589-595

2 Weiss JL, Malone FD, Emig D, et al; FASTER Research Consortium. Obesity, obstetric complications and cesarean delivery rate-a population-based screening study. Am J Obstet Gynecol 2004; 190(04):1091-1097

3 Galtier-Dereure F, Boegner C, Bringer J. Obesity and pregnancy: complications and cost. Am J Clin Nutr 2000;71(5, Suppl):1242S-1248S

4 Lashen H, Fear K, Sturdee DW. Obesity is associated with increased risk of first trimester and recurrent miscarriage: matched casecontrol study. Hum Reprod 2004;19(07):1644-1646

5 Salihu HM, Dunlop AL, Hedayatzadeh M, Alio AP, Kirby RS, Alexander GR. Extreme obesity and risk of stillbirth among black and white gravidas. Obstet Gynecol 2007;110(03):552-557

6 Boney CM, Verma A, Tucker R, Vohr BR. Metabolic syndrome in childhood: association with birth weight, maternal obesity, and gestational diabetes mellitus. Pediatrics 2005;115(03):e290-e296

7 Catalano PM, Farrell K, Thomas A, et al. Perinatal risk factors for childhood obesity and metabolic dysregulation. Am J Clin Nutr 2009;90(05):1303-1313

8 Hull HR, Dinger MK, Knehans AW, Thompson DM, Fields DA. Impact of maternal body mass index on neonate birthweight and body composition. Am J Obstet Gynecol 2008;198(04):416.e1-416.e6

9 Krakowiak P, Walker CK, Bremer AA, et al. Maternal metabolic conditions and risk for autism and other neurodevelopmental disorders. Pediatrics 2012;129(05):e1121-e1128

10 Edlow AG, Guedj F, Pennings JL, Sverdlov D, Neri C, Bianchi DW. Males are from Mars, and females are from Venus: sex-specific fetal brain gene expression signatures in a mouse model of maternal dietinduced obesity. Am JObstet Gynecol 2016;214(05):623.e1-623.e10

11 Howie GJ, Sloboda DM, Kamal T, Vickers MH. Maternal nutritional history predicts obesity in adult offspring independent of postnatal diet. J Physiol 2009;587(Pt 4):905-915

12 Volpato AM, Schultz A, Magalhães-da-Costa E, Correia ML, Águila MB, Mandarim-de-Lacerda CA. Maternal high-fat diet programs for metabolic disturbances in offspring despite leptin sensitivity. Neuroendocrinology 2012;96(04):272-284
13 Rowan JA, Hague WM, Gao W, Battin MR, Moore MP MiG Trial Investigators. Metformin versus insulin for the treatment of gestational diabetes. N Engl J Med 2008;358(19):2003-2015

14 Zoncu R, Efeyan A, Sabatini DM. mTOR: from growth signal integration to cancer, diabetes and ageing. Nat Rev Mol Cell Biol 2011;12(01):21-35

15 Chiswick C, Reynolds RM, Denison F, et al. Effect of metformin on maternal and fetal outcomes in obese pregnant women (EMPOWaR): a randomised, double-blind, placebo-controlled trial. Lancet Diabetes Endocrinol 2015;3(10):778-786

16 Syngelaki A, Nicolaides KH, Balani J, et al. Metformin versus Placebo in Obese Pregnant Women without Diabetes Mellitus. N Engl J Med 2016;374(05):434-443

17 Laplante M, Sabatini DM. mTOR signaling in growth control and disease. Cell 2012;149(02):274-293

18 Dowling RJ, Topisirovic I, Fonseca BD, Sonenberg N. Dissecting the role of mTOR: lessons from mTOR inhibitors. Biochim Biophys Acta 2010;1804(03):433-439

19 Howell JJ, Hellberg K, Turner M, et al. Metformin Inhibits Hepatic mTORC1 Signaling via Dose-Dependent Mechanisms Involving AMPK and the TSC Complex. Cell Metab 2017;25(02):463-471

20 Vora NL, Grace MR, Smeester L, et al. Targeted Multiplex Gene Expression Profiling to Measure High-Fat Diet and Metformin Effects on Fetal Gene Expression in a Mouse Model. Reprod Sci 2018 (e-pub ahead of print). Doi: 10.1177/1933719118786453

21 Emami Riedmaier A, Fisel P, Nies AT, Schaeffeler E, Schwab M. Metformin and cancer: from the old medicine cabinet to pharmacological pitfalls and prospects. Trends Pharmacol Sci 2013;34 (02):126-135

22 Schuler KM, Rambally BS, DiFurio MJ, et al. Antiproliferative and metabolic effects of metformin in a preoperative window clinical trial for endometrial cancer. Cancer Med 2015;4(02):161-173

23 Romero R, Erez O, Hüttemann M, et al. Metformin, the aspirin of the 21st century: its role in gestational diabetes mellitus, prevention of preeclampsia and cancer, and the promotion of longevity. Am J Obstet Gynecol 2017;217(03):282-302

24 Chandel NS, Avizonis D, Reczek CR, et al. Are metformin doses used in murine cancer models clinically relevant? Cell Metab 2016;23(04):569-570

25 Dowling RJ, Lam S, Bassi C, et al. Metformin pharmacokinetics in mouse tumors: implications for human therapy. Cell Metab 2016; 23(04):567-568

26 Magnuson B, Ekim B, Fingar DC. Regulation and function of ribosomal protein S6 kinase (S6K) within mTOR signalling networks. Biochem J 2012;441(01):1-21

$27 \mathrm{Yu}$ JS, Cui W. Proliferation, survival and metabolism: the role of $\mathrm{PI} 3 \mathrm{~K} / \mathrm{AKT} / \mathrm{mTOR}$ signalling in pluripotency and cell fate determination. Development 2016;143(17):3050-3060

28 Brown EJ, Beal PA, Keith CT, Chen J, Shin TB, Schreiber SL. Control of p70 s6 kinase by kinase activity of FRAP in vivo. Nature 1995; 377(6548):441-446

29 Corradetti MN, Guan KL. Upstream of the mammalian target of rapamycin: do all roads pass through mTOR? Oncogene 2006;25 (48):6347-6360

30 Gürke J, Schindler M, Pendzialek SM, et al. Maternal diabetes promotes mTORC1 downstream signalling in rabbit preimplantation embryos. Reproduction 2016;151(05):465-476

31 Roos S, Jansson N, Palmberg I, Säljö K, Powell TL, Jansson T. Mammalian target of rapamycin in the human placenta regulates leucine transport and is down-regulated in restricted fetal growth. J Physiol 2007;582(Pt 1):449-459 